\title{
Coronavirus and Football (Soccer): Why mid-day matches are much safer for players
}

Aliza Fedorenko ${ }^{1}$, Tomer Orevi ${ }^{1}$, Nadav Kashtan $^{1 \dagger}$

${ }^{1}$ Department of Plant Pathology and Microbiology, Robert H. Smith Faculty of Agriculture, Food, and Environment, Hebrew University of Jerusalem, Rehovot, Israel 76100

$\uparrow$ Corresponding author: Nadav Kashtan: nadav.kashtan@mail.huji.ac.il

\begin{abstract}
Safely resuming sporting events while the coronavirus is spreading is challenging - yet possible - if the science is taken into account. Two main ways the coronavirus can spread among football players is through air-suspended microdroplets (and possibly aerosols), and via contact with contaminated surfaces. Here we estimated virus survival in dried saliva droplets on a football pitch (i.e., on the grass) and on the ball itself, and compared these measures between mid-day and nighttime matches. We find, based on experiments with the enveloped phage Phi6 - a surrogate for SARS-Cov-2 - that while the virus survives reasonably well on both pitch and ball during a nighttime match ( $\sim 10 \%$ survival $)$, virtually no viruses survived the 90 minute duration of a mid-day match on a hot, sunny day. These results, taken together with studies reporting rapid deactivation of coronavirus in aerosols by sunlight, suggest that playing football in mid-day reduces the likelihood of transmission between players, and thus increases players' safety.
\end{abstract}

Keywords COVID-19; football; soccer; microbiology; microdroplets; saliva; SARS-CoV-2, sport; soccer; surfaces; transmission; viruses

While the novel coronavirus spreads around the globe, efforts are being made at a return to normalcy, including resuming sporting events. One of the most popular sports - football (soccer) - has recently restarted in some countries, yet without spectators. We currently do not know enough about the transmission routes of the virus to be able to estimate the risk of transmission between football players during a match ${ }^{1}$. One way to minimize this risk is by choosing the time of the day a match is played. According to our current understanding, the virus can be transmitted via air (via droplets and possibly aerosols) and through contact with contaminated surfaces. Two surfaces players are in frequent contact are the pitch (i.e. the grass), and the ball itself, that is frequently touched by hand as well. Here we sought to compare virus survival on the pitch (i.e., on the grass) and on the ball itself, between games played at night and at mid-day (Figure 1).

SARS-CoV-2 has been shown to survive on inanimate surfaces for several days ${ }^{2-4}$, and particularly in evaporated saliva microdroplets. Human saliva microdroplets expelled into the air through coughing, talking, and breathing, are considered a key source of transmission of the virus ${ }^{5,6}$. These microdroplets, ranging in size from a few micrometers to millimeters ${ }^{7-9}$ travel through the air, and some of them - larger ones in particular - settle on surfaces ${ }^{6}$. In addition, football players are known for their habit of frequently spitting on the pitch while playing. Thus, saliva droplets are constantly settling on both the pitch itself and the ball during a game. While dry deposits of saliva droplets on surfaces have been shown to protect viruses $^{10-12}$, their survival is affected, at least to some degree, by temperature and relative humidity (RH), with a typically higher survival rate in colder and dryer conditions. In addition, sunlight and UV radiation have been shown to rapidly deactivate the coronavirus ${ }^{13,14}$.

We thus sought to compare the virus survival in saliva droplets settled on the pitch (on grass) and on the ball, between a mid-day and nighttime matches. In our experiment, we simulated a match played on a warm, 


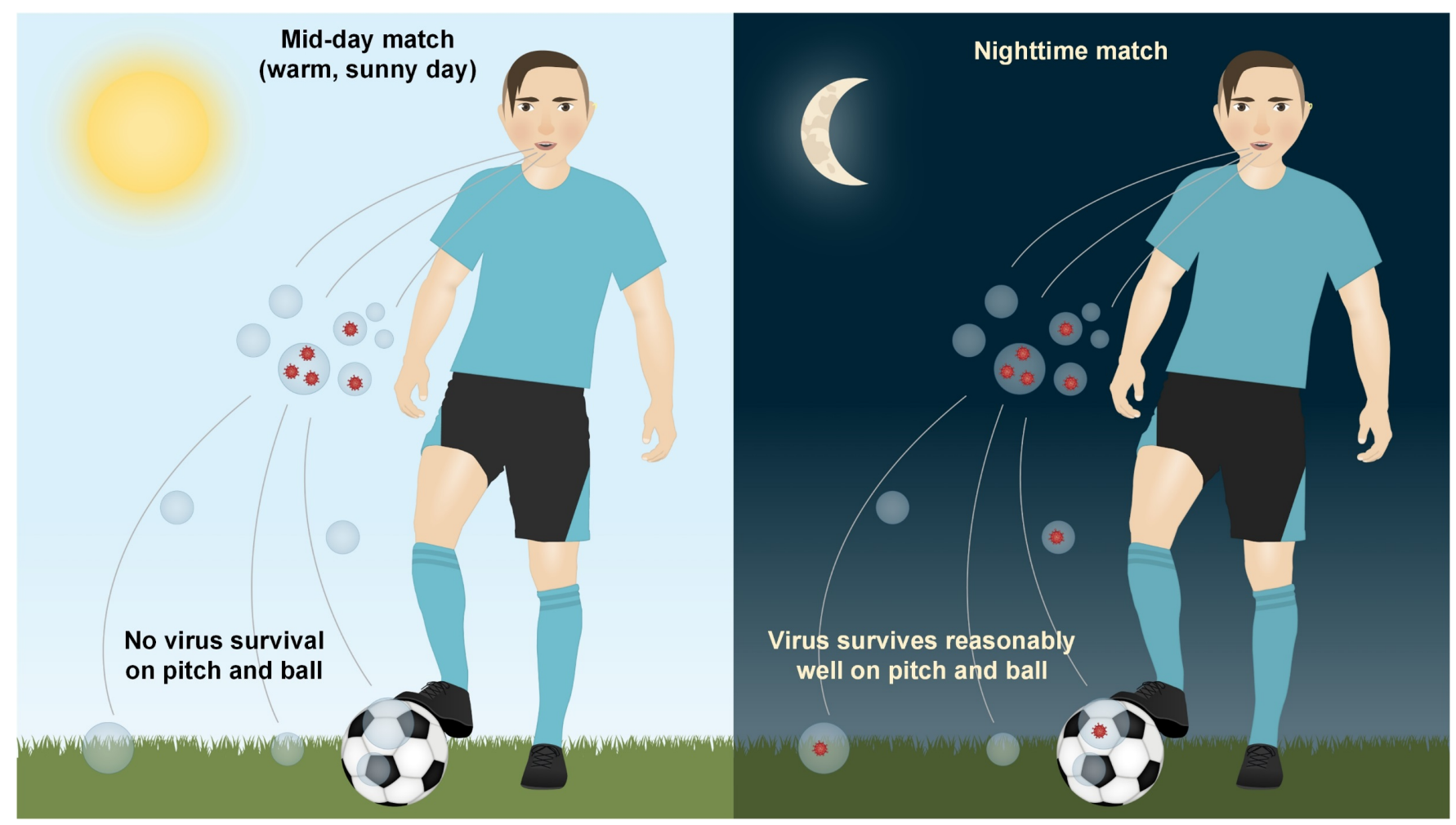

Figure 1 Virus survival on the pitch and on the ball is much lower in mid-day football matches in comparison to nighttime matches. Microdroplets and virus are not drawn to scale.

sunny day at 1 p.m., and a nighttime game, played at cooler temperatures, higher relative humidity, and with little to no sunlight at 8 p.m. (Figure 1).

As working with the coronavirus directly is complicated, we used the enveloped phage Phi6, which has been proposed as a good surrogate for SARS coronaviruses ${ }^{11,12,15-19}$. Phi6 is a dsRNA bacteriophage (a virus that infects bacteria) of the Cystoviridae family, that similarly to SARS-CoV-2, is enveloped by a lipid membrane, has spike proteins, and is of a similar size $(\sim 80-100 \mathrm{~nm})$.

To measure the survival of viable (i.e., infective) viruses, we suspended Phi6 phages in human saliva and deposited the sample on two surface types: a football, and grass. The deposits were then placed in an open field, exposed to sunlight and wind (similar to an open football field), and were monitored over the course of 24 hours at four time points: (i) at $\mathrm{t}=0$, as control; (ii) at $\mathrm{t}=105$ minutes, reflecting a full game's length (90 minutes +15 minutes break); (iii) at $\mathrm{t}=8$ hours; (iv) at $\mathrm{t}=24$ hours (next day match). To be able to compare virus decay between a mid-day and nighttime matches, two identical experiments were conducted, except that one began at 1:00 p.m., and the other at 8:00 p.m.

The viability of the retrieved samples was measured using a standard plaque assay (see Methods). The results were quite remarkable (Figure 2 ). In the mid-day match $\left(30^{\circ} \mathrm{C}, \mathrm{RH}=\sim 50 \%\right)$, virtually no virus survived the duration of a game, either on the grass or on the ball (Figure 2, dashed line). This was in contrast to the nighttime game, where viruses showed around tenfold decay $(\sim 10 \%$ of the viruses remained viable) within the duration of a game (Figure 2, solid line). In the nighttime match, the viruses were exposed to lower temperatures of around $22^{\circ} \mathrm{C}$, and higher $\mathrm{RH}$ levels of $\sim 80 \%$, and absence of sunlight. The zero survival in the mid-day match is thus likely due to the heat and sunlight. In the evening match, the balldeposited viruses decayed moderately overnight, dropping 3 orders of magnitude $(\sim 0.1 \%$ of viruses survived after 8 hours), while the viruses on the grass appeared more stable, with only minor decay (between $5-10 \%$ of the viruses remained viable at $\mathrm{t}=8$ hours). On both grass and ball, the saliva drops were not fully dry after 8 hours; however, they did dry completely before $t=24$ hours. No viable viruses were observed a $\mathrm{t}=24$ hours (Figure 2). This may not be that surprising, in light of the mid-day rapid deactivation of the 


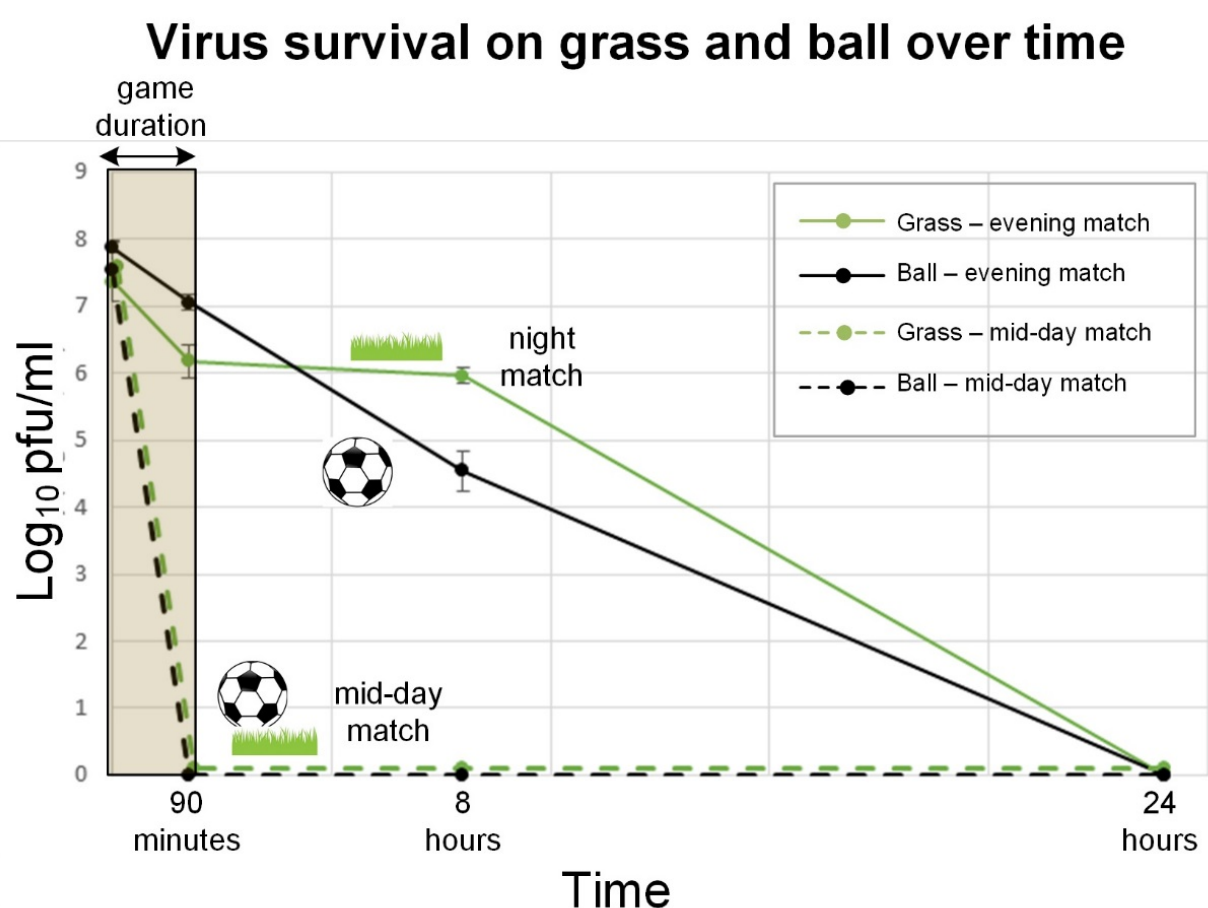

Figure 2 A comparison of virus survival dynamics between a nighttime and mid-day football matches. Viruses suspended within saliva droplets were deposited on the pitch (i.e. grass) and on the ball during the experiment and tracked for 24 hours. In the mid-day match no viruses survived the duration of a match $(90$ minutes +15 minutes break) both on the pitch and on the ball. Mean and Standard deviations are for duplicates $(n=2)$.

virus, as the nighttime viruses were exposed to sunlight and heat the following day (samples were left in the same location for the duration of the whole experiment).

In summary, our results indicate that in terms of players' safety, there is a significant advantage to playing during mid-day hours on hot sunny days, where sunlight and heat lead to rapid deactivation of the virus. Playing football during mid-day hours on a hot, sunny day is therefore likely to be much safer than spending time in the locker or shower rooms or on the bus before and after a game. We note that while our experiments were performed with a surrogate for SARS-CoV-2, we believe that the results would not change much for the coronavirus. In addition, the relative contribution of transmission via contact with contaminated surfaces is still unknown. Nonetheless, these results, together with other reports on rapid killing of the virus in aerosols by sunlight and UV radiation, suggest that playing football in mid-day reduces the risk of coronavirus transmission. We therefore suggest that, in warm and sunny weather, moving football games to mid-day hours is likely to increase players' safety.

\section{Methods}

Experimental setup: A stock solution of Phi6 bacteriophage $\left(\sim 10^{10} \mathrm{pfu} / \mathrm{ml}\right)$ was diluted 1:100 in human saliva (kindly donated by one of the co-authors) and immediately placed on either separate cut-pieces of a football ball made of synthetic leather, or on freshly cut grass. Both samples were placed on concave surfaces to maximize sample retrieval. $500 \mu$ ls of the phage-in-saliva solution were placed on each surface (in duplicates per sampling time-point), and all samples were placed in an open field at the beginning of each experiment. The samples were collected at the appointed time point (e.g., $\mathrm{t}=8$ hours) and resuspended in $\mathrm{SM}$ buffer $\left(100 \mathrm{mM} \mathrm{NaCl}, 8 \mathrm{mM} \mathrm{MgSO} 4 \times 7 \mathrm{H}_{2} \mathrm{O}, 50 \mathrm{mM}\right.$ Tris-Cl $\mathrm{pH} 7.5,0.01 \% \mathrm{w} / \mathrm{v}$ Gelatin) to a final volume of $1 \mathrm{ml}$, then serially diluted and measured through drop plaque assay.

Plaque Assay: Plates containing a bottom agar layer were poured in advance (TSB with $15 \mathrm{~g} / 1$ agar, $5 \mathrm{mM}$ $\mathrm{MgSO}_{4}$ and $5 \mathrm{mM} \mathrm{CaCl}_{2}$ ). On the day of the experiment, an overnight bacterial host culture (P. syringae) grown at $28^{\circ} \mathrm{C}$ with shaking, was diluted 1:50 into fresh medium and shake incubated until it reached $\mathrm{OD}_{600}$ of 0.3 . Meanwhile, the top agar (TSB with $7.5 \mathrm{~g} / 1$ agar, $5 \mathrm{mM} \mathrm{MgSO}_{4}$, and $5 \mathrm{mM} \mathrm{CaCl}_{2}$ ) was melted and kept in a $55^{\circ} \mathrm{C}$ water bath. The bacterial culture was combined with the top agar at a ratio of 
1:40, and poured on top of the bottom layer. The re-suspended phages were serially diluted in SM buffer, and after the top agar had solidified, $10 \mu 1$ were pipetted and spread on the agar plates and left open until dry and incubated at $28^{\circ} \mathrm{C}$ overnight.

\section{Acknowledgements}

We thank Dr. Yael Helman for fruitful discussions that led to the idea of comparing between a mid-day and nighttime matches.

\section{References}

1 Knudsen, N. S., Thomasen, M. M. D. \& Andersen, T. B. Spread of virus during soccer matches. medRxiv (2020).

2 van Doremalen, N. et al. Aerosol and Surface Stability of SARS-CoV-2 as Compared with SARSCoV-1. New England Journal of Medicine (2020).

3 Chin, A. et al. Stability of SARS-CoV-2 in different environmental conditions. medRxiv (2020).

4 Liu, Y. et al. Stability of SARS-CoV-2 on environmental surfaces and in human excreta. medRxiv, doi:https://doi.org/10.1101/2020.05.07.20094805 (2020).

5 Organization, W. H. Modes of transmission of virus causing COVID-19: implications for IPC precaution recommendations: scientific brief, 27 March 2020. (World Health Organization, 2020).

6 Prather, K. A., Wang, C. C. \& Schooley, R. T. Reducing transmission of SARS-CoV-2. Science (2020).

7 Dbouk, T. \& Drikakis, D. On coughing and airborne droplet transmission to humans. Physics of Fluids 32, 053310, doi: https://doi.org/10.1063/5.0011960 (2020).

8 Duguid, J. The size and the duration of air-carriage of respiratory droplets and droplet-nuclei. Epidemiology \& Infection 44, 471-479 (1946).

9 Yang, W. \& Marr, L. C. Dynamics of airborne influenza A viruses indoors and dependence on humidity. PloS one 6 (2011).

10 Santosh Kumar, S., Shao, S., Li, J., He, Z. \& Hong, J. Droplet evaporation residue indicating SARS-COV-2 survivability on surfaces. arXiv, arXiv: 2005.12262 (2020).

11 Fedorenko Aliza, Grinberg Maor, Orevi Tomer. \& Nadav., K. Virus survival in evaporated saliva microdroplets deposited on inanimate surfaces. bioRxiv, https://doi.org/10.1101/2020.1106.1115.152983 (2020).

12 Vejerano, E. P. \& Marr, L. C. Physico-chemical characteristics of evaporating respiratory fluid droplets. Journal of The Royal Society Interface 15, 20170939 (2018).

13 Schuit, M. et al. Airborne SARS-CoV-2 is Rapidly Inactivated by Simulated Sunlight. The Journal of Infectious Diseases (2020).

14 García de Abajo, F. J. et al. Back to Normal: An Old Physics Route to Reduce SARS-CoV-2 Transmission in Indoor Spaces. ACS Nano (2020).

15 Aquino de Carvalho, N., Stachler, E. N., Cimabue, N. \& Bibby, K. Evaluation of Phi6 persistence and suitability as an enveloped virus surrogate. Environmental science \& technology 51, 86928700 (2017).

16 Ford, B. E. Pseudomonas Bacteriophage Phi6 as a Model for Virus Emergence. (2015).

17 Prussin, A. J. et al. Survival of the enveloped virus Phi6 in droplets as a function of relative humidity, absolute humidity, and temperature. Appl. Environ. Microbiol. 84, e00551-00518 (2018).

18 Turgeon, N., Toulouse, M.-J., Martel, B., Moineau, S. \& Duchaine, C. Comparison of five bacteriophages as models for viral aerosol studies. Appl. Environ. Microbiol. 80, 4242-4250 (2014).

19 Adcock, N. J. et al. The use of bacteriophages of the family Cystoviridae as surrogates for H5N1 highly pathogenic avian influenza viruses in persistence and inactivation studies. Journal of Environmental Science and Health, Part A 44, 1362-1366 (2009). 